JINR-P2-82-900

\title{
Anomalous dimensions of quark masses in the three-loop approximation
}

\author{
O.V. Tarasov \\ Joint Institute for Nuclear Research, Dubna, Russia
}

\begin{abstract}
The results of calculation of the three-loop radiative correction to the renormalization constant of fermion masses for non-abelian gauge theory interacting with fermions are presented. Dimensional regularization and the t'Hooft-Veltman minimal subtraction scheme are used. The method of calculation is described in detail. The renormalization group function $\gamma_{m}$ determining the behavior of the effective mass of fermions is presented. The anomalous dimensions of fermions for QED and QCD up to three loops are given. All calculations were performed on a computer with the help of the SCHOOONSCHIP system for analytical manipulations.
\end{abstract}


The effects determined by the nonzero quark and lepton masses in quantum chromodynamics, the standard $S U(2) \times U(1)$ model, usual and supersymmetric grand unified theories are now attracting now more and more attention. Considerable efforts were devoted to determining the mass of the Higgs scalar particle from the LEP experiments. The threshold effects [1], the light quark masses [2] and the value of the ratio $m_{b} / m_{\tau}$ in the frame of the grand unified theories [3] are usually investigated by the renormalization group (RG) method [4]. Application of the RG allows one to sum the leading momentum-dependent logarithms to all orders in terms of the limited number of the RG parameters (beta functions and anomalous dimensions) which are calculated in the framework of perturbation theory.

The most characteristic feature of the mass account is large contributions of the higher orders in perturbation theory. For example, the two-loop corrections to the ratio $m_{b} / m_{\tau}$ [3] are $20 \%$ from the leading contribution. In the $S U(5)$ grand unified theory from two-loop analysis it was concluded [3] that the number of flavors cannot exceed six. The one - loop approximation gives a less rigid restriction- the number of flavors may be less than eight. The next approximations - the three loop and higher, can also change this restriction. Higher corrections to the renormalization group functions depend more strongly on the number of quarks.

We consider the non-Abelian gauge theory with fermions belonging to the representation $R$ of the gauge group $\mathrm{G}$ :

$$
\begin{gathered}
L=-\frac{1}{4} G_{\mu \nu}^{a} G_{\mu \nu}^{a}-\frac{1}{2 \alpha}\left(\partial_{\mu} A_{\mu}^{a}\right)^{2}-\partial_{\mu} \bar{\eta}^{a} \partial_{\mu} \eta^{a}+g f^{a b c} \bar{\eta}^{a} A_{\mu}^{b} \partial_{\mu} \eta^{c}+i \sum_{l=1}^{f} \bar{\psi}_{i}^{l} \hat{D} \psi_{i}^{l}-\sum_{l=1}^{f} m_{l} \bar{\psi}_{i}^{l} \psi_{i}^{l}, \\
G_{\mu \nu}^{a}=\partial_{\mu} A_{\nu}^{a}-\partial_{\nu} A_{\mu}^{a}+g f^{a b c} A_{\mu}^{b} A_{\nu}^{c} \\
D_{\mu} \psi_{i}^{l}=\partial_{\mu} \psi_{i}^{l}-i g R_{i j}^{a} \psi_{j}^{l} A_{\mu}^{a} .
\end{gathered}
$$

Here $\eta^{a}$ is the ghost field, $\alpha$ is the gauge parameter and $f^{a b c}$ are totally antisymmetric structure constants of the gauge group $G$. The indices of the fermion field $\psi_{i}^{m}$ specify color $(i)$ and flavor $(m)$, respectively. The matrices $R^{a}$ obey the following relations:

$$
\begin{gathered}
{\left[R^{a}, R^{b}\right]_{-}=i f^{a b c} R^{c}, \quad f^{a c d} f^{b c d}=C \delta^{a b},} \\
R^{a} R^{a}=C_{F} I, \quad \operatorname{tr}\left(R^{a} R^{b}\right)=t \delta^{a b} .
\end{gathered}
$$

In particular, the values of group invariants $C, C_{F}$ and $T$ in the fundamental (quark) representation of $S U(N)$ are:

$$
C=N, \quad C_{F}=\frac{N^{2}-1}{2 N}, \quad t=\frac{1}{2} .
$$

In this paper we adopt the renormalization prescription by 't Hooft [5] the so-called "minimal subtraction scheme" (MS), which by definition subtracts only pole parts in $\varepsilon$ from a given diagram. The renormalization constant $Z_{\Gamma}$ relating the dimensionally regularized 1PI Green function with the renormalized one

$$
\Gamma_{R}\left(\frac{Q^{2}}{\mu^{2}}, h, m, \alpha\right)=\lim _{\varepsilon \rightarrow 0} Z_{\Gamma}\left(\frac{1}{\varepsilon}, h, \alpha\right) \Gamma\left(Q^{2}, h_{B}, m_{B}, \alpha_{B}, \varepsilon\right),
$$


looks in this scheme like

$$
Z_{\Gamma}\left(\frac{1}{\varepsilon}, h, \alpha\right)=1+\sum_{\nu=1}^{\infty} c_{\Gamma}^{(\nu)}(h, \alpha) \frac{1}{\varepsilon^{\nu}},
$$

with $\varepsilon=(4-n) / 2, n$ being the space-time dimension, $h=g^{2} /(4 \pi)^{2}$ and $\mu$ is the renormalization parameter with the dimension of mass. The relationship between the bare charge $h_{B}^{2}$ and the renormalized one includes the product of appropriate $Z$ 's. The most convenient choice of $Z$ 's is as follows:

$$
h_{B}=\left(\mu^{2}\right)^{\varepsilon} h \widetilde{Z}_{1}^{2} Z_{3}^{-1} \widetilde{Z}_{3}^{-2}=\left(\mu^{2}\right)^{\varepsilon}\left[h+\sum_{\nu=1}^{\infty} a^{(\nu)}(h) \frac{1}{\varepsilon^{\nu}}\right] \text {. }
$$

Here $\widetilde{Z}_{1}$ is the renormalization constant of the ghost-ghost-gluon vertex, $Z_{3}$ and $\widetilde{Z}_{3}$ being those of the inverse gluon and ghost propagators, respectively. Bare parameters $m_{B}$ and $\alpha_{B}$ are connected with the renormalized ones as:

$$
\begin{aligned}
& m_{B}=Z_{m} m=m\left[1+\sum_{\nu=1}^{\infty} b^{(\nu)}(h) \frac{1}{\varepsilon^{\nu}}\right], \\
& \alpha_{B}=Z_{3} \alpha=\alpha\left[1+\sum_{\nu=1}^{\infty} d^{(\nu)}(h, \alpha) \frac{1}{\varepsilon^{\nu}}\right] .
\end{aligned}
$$

In Ref. [6] it was shown that $a^{(\nu)}(h)$ and $b^{(\nu)}(h)$ in the $\overline{M S}$ renormalization scheme are gauge independent. This gives us a possibility to use the simplest gauge for calculation. We choose to work in the Feynman gauge $\alpha=1$ throughout this paper.

The Green function $\Gamma_{R}\left(\frac{Q^{2}}{\mu^{2}}, h^{2}, m, \alpha\right)$ in the $\overline{M S}$ scheme satisfies the renormalization group equation [5], [7]:

$$
\begin{aligned}
\left\{Q^{2} \frac{\partial}{\partial Q^{2}}-\beta(h) \frac{\partial}{\partial h}\right. & +\left[1-\gamma_{m}(h)\right] m \frac{\partial}{\partial m} \\
& \left.-\delta(h, \alpha) \alpha \frac{\partial}{\partial \alpha}-\gamma_{\Gamma}(h, \alpha)\right\} \Gamma_{R}\left(\frac{Q^{2}}{\mu^{2}}, h, m, \alpha\right)=0 .
\end{aligned}
$$

Here

$$
\begin{aligned}
& \beta(h)=\left.\mu^{2} \frac{\partial h}{\partial \mu^{2}}\right|_{h_{B}, m_{B}, \alpha_{B}-\text { fixed }}, \\
& \gamma_{m}(h)=\left.\mu^{2} \frac{\partial \ln m}{\partial \mu^{2}}\right|_{h_{B}, m_{B}, \alpha_{B}-\text { fixed }}, \\
& \delta(h, \alpha)=\left.\mu^{2} \frac{\partial \ln \alpha}{\partial \mu^{2}}\right|_{h_{B}, m_{B}, \alpha_{B}-\text { fixed }}, \\
& \gamma_{\Gamma}(h, \alpha)=-\left.\mu^{2} \frac{\partial \ln Z_{\Gamma}}{\partial \mu^{2}}\right|_{h_{B}, m_{B}, \alpha_{B}-\text { fixed }} .
\end{aligned}
$$

The functions $\beta(h), \gamma_{m}(h), \delta(h, \alpha)$ and $\gamma_{\Gamma}(h, \alpha)$ can be expressed in terms of the coefficients in front of $\varepsilon^{-1}$ in the decompositions of the corresponding renormalization 
constants $Z$ [5], [7]:

$$
\begin{aligned}
& \beta(h)=\left(h \frac{\partial}{\partial h}-1\right) a^{(1)}(h) \\
& =h\left[2 \widetilde{\gamma}_{1}(h, \alpha)-\gamma_{3}(h, \alpha)-2 \widetilde{\gamma}_{3}(h, \alpha)\right]=-\beta_{1} h^{2}-\beta_{2} h^{3}-\beta_{3} h^{4}-\cdots, \\
& \gamma_{m}(h)=h \frac{\partial b^{(1)}(h)}{\partial h}=-\gamma_{1} h-\gamma_{2} h^{2}-\gamma_{3} h^{3}-\cdots, \\
& \delta(h, \alpha)=h \frac{\partial d^{(1)}(h)}{\partial h}=\gamma_{3}(h, \alpha), \\
& \gamma_{\Gamma}(h, \alpha)=h \frac{\partial c^{(1)}(h)}{\partial h},
\end{aligned}
$$

where $\widetilde{\gamma}_{1}(h)$ is the anomalous dimension of the ghost-ghost-gluon vertex, $\gamma_{3}$ and $\widetilde{\gamma}_{3}$ are anomalous dimensions of the gluon and ghost propagators respectively. The coefficients in front of $1 / \varepsilon^{\nu}(\nu \geq 2)$ for the renoramlization constants $Z_{\Gamma}$ and $Z_{m}$ are related to $c^{(1)}$ and $b^{(1)}$ by [5], [7]:

$$
\begin{aligned}
& \left\{\beta(h) \frac{\partial}{\partial h}+\gamma_{3}(h, \alpha) \alpha \frac{\partial}{\partial \alpha}+\gamma_{\Gamma}(h, \alpha)\right\} c^{(\nu)}(h, \alpha)=h \frac{\partial}{\partial h} c^{(\nu+1)}(h, \alpha), \\
& \left\{\gamma_{m}(h)+\beta(h) \frac{\partial}{\partial h}\right\} b^{(\nu)}(h)=h \frac{\partial}{\partial h} b^{(\nu+1)}(h) .
\end{aligned}
$$

These relationships were used to partially verify our calculations.

The goal of this paper is to calculate the three-loop approximation for $\gamma_{m}(h)$. The two-loop calculations were presented in Refs. [8]. To find $\gamma_{m}(h)$, it is convenient to use the relation $\gamma_{m}(h)=-\gamma_{2}(h, \alpha)+\gamma_{\bar{\psi} \psi}(h, \alpha)$ that follows from the equation

$$
Z_{m}=Z_{\bar{\psi} \psi} Z_{2}^{-1}
$$

where

$$
\gamma_{\bar{\psi} \psi}(h, \alpha)=-\left.\mu^{2} \frac{\partial}{\partial \mu^{2}} \ln Z_{\bar{\psi} \psi}\right|_{h_{B}, m_{B}, \alpha_{B}-\text { fixed }},
$$

and $Z_{\bar{\psi} \psi}$ is the renormalization constant of the two-point fermion Green function with the insertion $\bar{\psi}(y) \psi(y)$, i.e

$$
\left\langle\bar{\psi}(x) \psi(0) \int d^{n} y \bar{\psi}(y) \psi(y)\right\rangle .
$$

According to the method described in [9], for the renormalization constants $Z_{\bar{\psi} \psi}$ and $Z_{2}$ we shall use the following representation:

$$
\begin{aligned}
& Z_{\bar{\psi} \psi}=1-K R^{\prime} \Gamma_{\bar{\psi} \psi}, \\
& Z_{2}^{-1}=1-K R^{\prime} \Gamma_{2} .
\end{aligned}
$$

Here $K$ is an operator that removes regular in $\varepsilon$ terms

$$
K \sum_{\nu} b_{\nu} \varepsilon^{\nu}=\sum_{\nu<0} b_{\nu} \varepsilon^{\nu}
$$


and $R^{\prime}$ is the $R$ operation for the minimal subtraction scheme without the last subtraction applied to the Green function $\Gamma$, i.e

$$
R=(1-K) R^{\prime}
$$

We apply $K$ and $R^{\prime}$ operations to the diagrams of the following type:

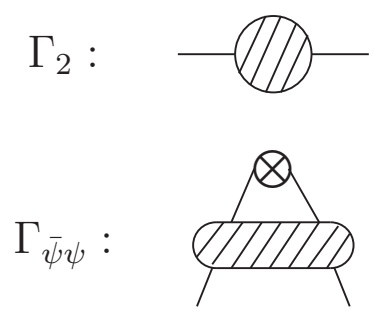

where $-\mathbb{Q}$ - corresponds to the insertion $\int \bar{\psi}(y) \psi(y) d^{n} y$.

As an illustrative example we consider calculation of the contribution to $Z_{\bar{\psi} \psi}$ from one of the three-loop diagrams. For example,

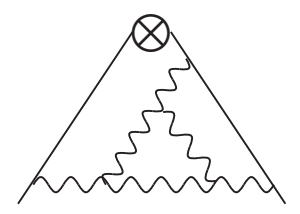

The $R^{\prime}$ for this diagram with the combinatorial factor and appropriate counterterms can schematically be represented as:

$$
\text { KR }
$$

Substituting expressions for the one- and two- loop diagrams and multiplying them by appropriate $K R^{\prime}$, we get for the diagram, counterterms with two- and one- loop subdiagrams the following expression: 


$$
\begin{aligned}
& =\left[\frac{13}{4 \varepsilon^{3}}+\frac{70}{3 \varepsilon^{2}}+\frac{1427}{12 \varepsilon}-\frac{113}{4 \varepsilon} \zeta(3)\right] C_{F} C^{2} \\
& +\left[\frac{39}{4 \varepsilon^{3}}+\frac{19}{4 \varepsilon^{2}}+\frac{231}{16 \varepsilon}\right] C_{F} C^{2} \\
& -\left[\frac{39}{4 \varepsilon^{3}}+\frac{299}{8 \varepsilon^{2}}+\frac{975}{8 \varepsilon}-\frac{117}{4 \varepsilon} \zeta(3)\right] C_{F} C^{2} \\
& =\left[\frac{13}{4 \varepsilon^{3}}-\frac{223}{24 \varepsilon^{2}}+\frac{551}{48 \varepsilon}+\frac{1}{\varepsilon} \zeta(3)\right] C_{F} C^{2} .
\end{aligned}
$$

All calculations were performed on the CDC-6500 computer using the algebraic manipulation system SCHOONSCHIP [10]. In Appendix, we present contributions to $Z_{2}^{-1}$ and $Z_{\bar{\psi} \psi}$ from diagrams with one- and two- loop insertions, factorized diagrams and 1PI diagrams. At the three loop level we get:

$$
\begin{aligned}
Z_{2}^{-1}= & 1+\frac{1}{\varepsilon} h C_{F}+\left[\frac{1}{\varepsilon^{2}}\left(\frac{1}{2} C_{F}-C\right)+\frac{1}{\varepsilon}\left(\frac{17}{4} C-t f-\frac{3}{4} C_{F}\right)\right] h^{2} C_{F} \\
& +\left[\frac{1}{\varepsilon^{3}}\left(\frac{7}{4} C^{2}-\frac{1}{3} C t f-C C_{F}+\frac{1}{6} C_{F}^{2}\right)\right. \\
& -\frac{1}{\varepsilon^{2}}\left(\frac{104}{9} C^{2}-\frac{56}{9} C t f+\frac{8}{9} t^{2} f^{2}-\frac{73}{12} C C_{F}+\frac{5}{3} C_{F} t f+\frac{3}{4} C_{F}^{2}\right) \\
& -\frac{1}{\varepsilon}\left(\frac{1301}{108} C t f+\frac{143}{12} C C_{F}-\frac{10559}{432} C^{2}-\frac{20}{27} t^{2} f^{2}\right. \\
Z_{m}= & \left.\left.-3 C_{F} t f-\frac{1}{2} C_{F}^{2}-4 C C_{F} \zeta(3)+\frac{5}{2} C^{2} \zeta(3)\right)\right] h^{3} C_{F}, \\
+ & {\left[\frac{1}{\varepsilon^{3}}\left(\frac{88}{9} C t f-\frac{1}{\varepsilon^{2}}\left(\frac{11}{2} C-2 t f+\frac{9}{2} C_{F}\right)+\frac{1}{\varepsilon}\left(-\frac{97}{12} C+\frac{5}{3} t f-\frac{3}{4} C_{F}\right)\right] h^{2} C_{F}-\frac{121}{9} C^{2}-\frac{16}{9} t^{2} f^{2}+6 C_{F} t f-\frac{9}{2} C_{F}^{2}\right) } \\
+ & \frac{1}{\varepsilon^{2}}\left(-\frac{484}{27} C t f+\frac{313}{12} C C_{F}+\frac{1679}{54} C^{2}+\frac{40}{27} t^{2} f^{2}-\frac{29}{3} C_{F} t f+\frac{9}{4} C_{F}^{2}\right) \\
+ & \frac{1}{\varepsilon}\left(\frac{556}{81} C t f+\frac{43}{4} C C_{F}-\frac{11413}{324} C^{2}+\frac{140}{81} t^{2} f^{2}+\frac{46}{3} C_{F} t f\right. \\
& \left.\left.\quad-\frac{43}{2} C_{F}^{2}+16 \zeta(3) t f\left(C-C_{F}\right)\right)\right] h^{3} C_{F} .
\end{aligned}
$$

Using (11) and (3) we get

$$
\begin{gathered}
\gamma_{m}(h)=-3 C_{F} h-\left(\frac{97}{6} C-\frac{10}{3} t f+\frac{3}{2} C_{F}\right) C_{F} h^{2}+\left(\frac{556}{27} C t f+\frac{129}{4} C C_{F}\right. \\
\left.-\frac{11413}{108} C^{2}+\frac{140}{27} t^{2} f^{2}+46 C_{F} t f-\frac{129}{2} C_{F}^{2}+48 \zeta(3) t f\left(C-C_{F}\right)\right) h^{3} C_{F},
\end{gathered}
$$




$$
\begin{gathered}
\gamma_{2}(h, 1)=-C_{F} h-\left(\frac{17}{2} C-2 t f-\frac{3}{2} C_{F}\right) C_{F} h^{2}-\left(\frac{10559}{144} C^{2}-\frac{1301}{36} C t f\right. \\
\left.-\frac{143}{4} C C_{F}+\frac{20}{9} t^{2} f^{2}+3 C_{F} t f+\frac{3}{2} C_{F}^{2}+12 C C_{F} \zeta(3)-\frac{15}{2} C^{2} \zeta(3)\right) h^{3} C_{F} .
\end{gathered}
$$

At the two loop level our results coincide with that presented in [8]. Unlike the $\beta$ function, the three-loop contribution to $\gamma_{m}$ depens on the Riemann function $\zeta(3)=$ $\sum_{l=1}^{\infty} 1 / l^{3}$. The coefficient in $\gamma_{m}$ proportional to $C_{F} t^{2} f^{2}$ coincides with that predicted in [11]. For the quantum chromodynamics $\left(S U(3)\right.$ gauge group with $C=3, C_{F}=4 / 3$, $t=1 / 2)$ we have:

$$
\begin{gathered}
\gamma_{m_{i}}(h)=-4 h-\left(\frac{202}{3}-\frac{20}{9} f\right) h^{2}-\left(\frac{3747}{3}-\frac{2216}{27} f-\frac{140}{81} f^{2}\right) h^{3}+\frac{160}{3} \zeta(3) f h^{3}, \\
\gamma_{2}(h, 1)=-\frac{4}{3} h-\left(\frac{97}{3}+\frac{4}{3} f\right) h^{2}-\left(\frac{24941}{36}-\frac{1253}{18} f+\frac{20}{27} f^{2}-26 \zeta(3)\right) h^{3} .
\end{gathered}
$$

For completeness we present also expression for the $\beta$ - function [12]:

$$
\beta(h)=-\left(11-\frac{2}{3} f\right) h^{2}-\left(102-\frac{38}{3} f\right) h^{3}-\left(\frac{2857}{2}-\frac{5033}{18} f+\frac{325}{54} f^{2}\right) h^{4} .
$$

From (4), one can easily get $\gamma_{2}$ and $\gamma_{m}$ for the quantum electrodynamics, by setting $C=0, C_{F}=1, t f=1$ :

$$
\begin{aligned}
\gamma_{m}(h) & =-3 h+\frac{11}{6} h^{2}-\frac{719}{54} h^{3}-48 \zeta(3) h^{3}, \\
\gamma_{2}(h) & =-h+\frac{7}{2} h^{2}-\frac{121}{18} h^{3} .
\end{aligned}
$$

The solutions of the renormalization group equation (2) in the t'Hooft scheme are expressed in terms of the effective parameters $\bar{h}, \bar{m}, \bar{\alpha}$ determined by the system of equations:

$$
\begin{aligned}
& \frac{d \bar{h}}{d l}=\beta(\bar{h}), \quad l=\ln \frac{Q^{2}}{\mu^{2}}, \\
& \frac{d \ln \bar{\alpha}}{d l}=\delta(\bar{h}, \bar{\alpha}), \\
& \frac{d \ln \bar{m}_{i}}{d l}=\gamma_{m_{i}}(\bar{h}) .
\end{aligned}
$$

The expression for $\bar{h}(L)$ up to three loops was given in [12]:

$$
\begin{aligned}
\bar{h}(L) & =\frac{1}{\beta_{1} L}-\frac{\beta_{2}}{\beta_{1}^{3}} \frac{\ln L}{L^{2}}+\frac{\beta_{2}^{2}\left(\ln ^{2} L-\ln L\right)}{\beta_{1}^{5} L^{3}}+\frac{\beta_{3} \beta_{1}-\beta_{2}^{2}}{\beta_{1}^{5} L^{3}} \\
& -\frac{\beta_{2}^{3} \ln ^{3} L}{\beta_{1}^{7} L^{4}}+\frac{5 \beta_{2}^{3} \ln ^{2} L}{2 \beta_{1}^{7} L^{4}}+\frac{\left(-3 \beta_{1} \beta_{2} \beta_{3}+2 \beta_{2}^{3}\right) \ln L}{\beta_{1}^{7} L^{4}}+O\left(\frac{1}{L^{4}}\right)
\end{aligned}
$$


Here $L=\ln \frac{Q^{2}}{\Lambda^{2}} \equiv \int \frac{d x}{\beta(x)}+\ln \frac{Q^{2}}{\mu^{2}}$. An arbitrary integration constant is chosen to cancel the term with $1 / L^{2}$ in the decomposition of $\bar{h}(L)$.

The solution for $\bar{m}_{i}$ is:

$$
\begin{aligned}
& \bar{m}_{i}(L)=\hat{m}_{i}(L)^{-\frac{\gamma_{1}}{\beta_{1}}}\left\{1+\frac{\left(\gamma_{2} \beta_{1}-\beta_{2} \gamma_{1}\right)}{\beta_{1}^{3} L}-\frac{\beta_{2} \gamma_{1}}{\beta_{1}^{3}} \frac{\ln L}{L}\right. \\
& +\frac{1}{2 \beta_{1}^{6}}\left(\beta_{1}^{3} \gamma_{3}+\beta_{1}^{2} \gamma_{2}^{2}-\beta_{1}^{2} \beta_{2} \gamma_{2}+\beta_{1}^{2} \beta_{3} \gamma_{1}-\beta_{1} \beta_{2}^{2} \gamma_{1}-2 \beta_{1} \beta_{2} \gamma_{1} \gamma_{2}+\beta_{2}^{2} \gamma_{1}^{2}\right) \frac{1}{L^{2}} \\
& \left.+\frac{1}{\beta_{1}^{6}}\left(\beta_{2}^{2} \gamma_{1}^{2}-\beta_{1}^{2} \beta_{2} \gamma_{2}-\beta_{1} \beta_{2} \gamma_{1} \gamma_{2}\right) \frac{\ln L}{L^{2}}+\frac{1}{2 \beta_{1}^{6}}\left(\beta_{1} \beta_{2}^{2} \gamma_{1}+\beta_{2}^{2} \gamma_{1}^{2}\right) \frac{\ln ^{2} L}{L^{2}}\right\} .
\end{aligned}
$$

At the present time $\delta(h, \alpha)$ in an arbitrary gauge at the three-loop level is not known. But in the MS scheme physical quantities depend only on $\bar{h}$, so for them we can use the renormalization group at the three-loop level.

The most interesting application of our results can be the $m_{b} / m_{\tau}$ ratio in the $S U(5)$ grand unified theory and calculation of $\gamma_{m}(h)$ at three loop level in supersymmetric theories [12.

Acknowledgments

I am very grateful to D.V.Shirkov, A.L. Kataev and D.I.Kazakov for valuable discussions.

The present text was published in 1982 as a JINR Communication, JINR-P2-82-900.

\section{Appendix}

The three-loop 1PI diagrams of the fermion propagator.
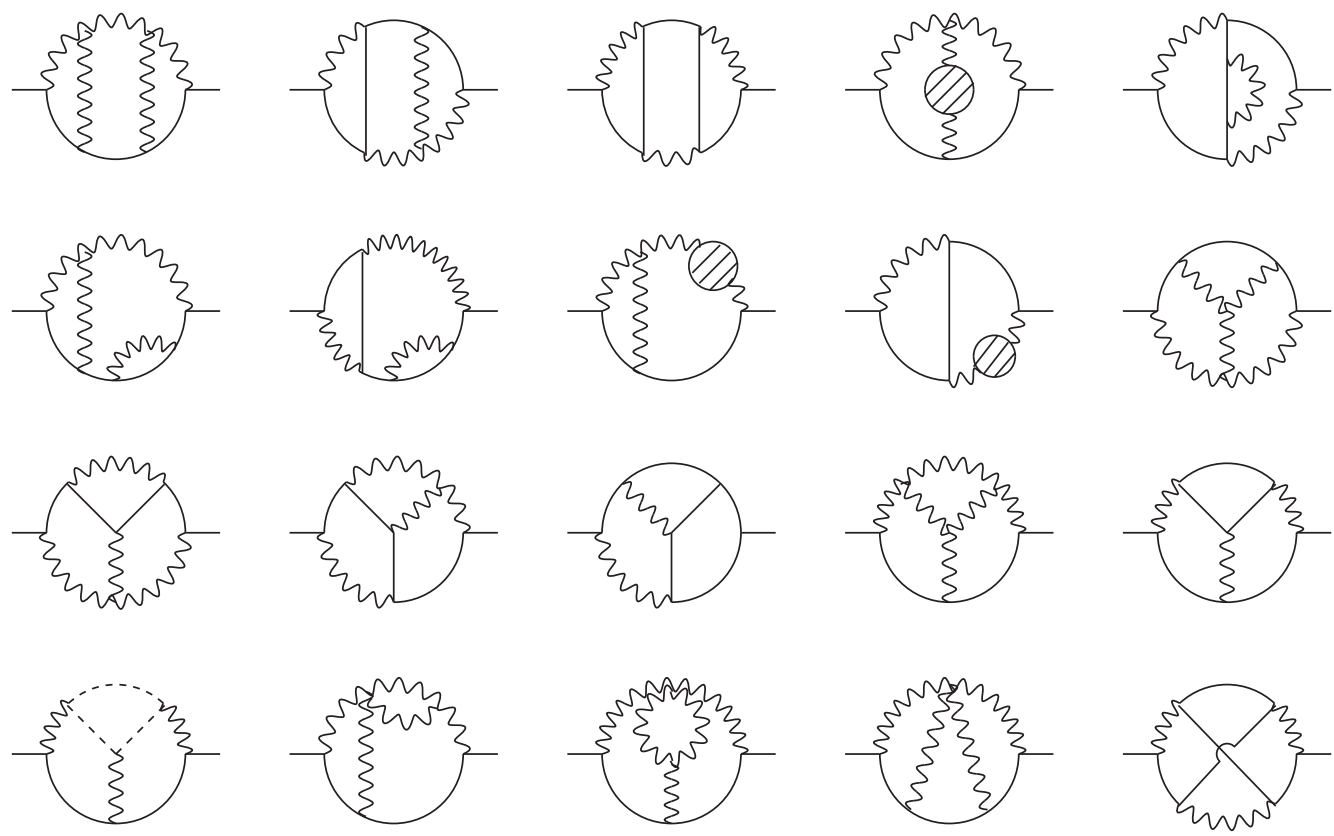

Fig.1 


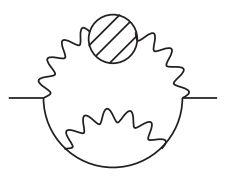

a)

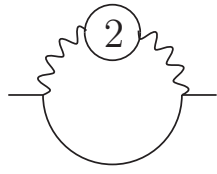

b)

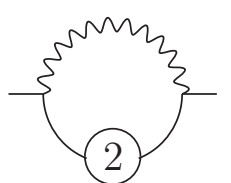

c)

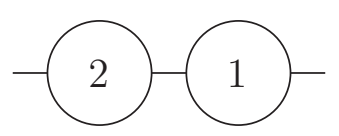

d)

Fig.2.

Fig.1.

The contributions to $Z_{2}^{-1}$ from different sets of the diagrams $\left(K R^{\prime}\right)$ :

$$
\begin{aligned}
& -\frac{C_{F}}{\varepsilon^{3}}\left[-\frac{7}{4} C^{2}-\frac{4}{3} C C_{F}+\frac{1}{3} C t f\right] \\
& -\frac{C_{F}}{\varepsilon^{2}}\left[\frac{219}{24} C^{2}+\frac{35}{6} C C_{F}-\frac{4}{3} C_{F}-\frac{19}{6} C t f-\frac{4}{3} C_{F} t f\right] \\
& -\frac{C_{F}}{\varepsilon}\left[-\frac{233}{12} C^{2}+\frac{17}{2} C C_{F}-\frac{7}{12} C_{F}^{2}+\frac{43}{6} C t f-\frac{7}{3} C_{F} t f\right]-\frac{C_{F}}{\varepsilon} C \zeta(3)\left(\frac{5}{2} C-2 C_{F}\right) .
\end{aligned}
$$

Fig.2a:

$$
\frac{C_{F}^{2}}{\varepsilon^{2}}\left(\frac{5}{12} C-\frac{1}{3} t f\right)+\frac{C_{F}^{2}}{\varepsilon}\left(\frac{3}{8} C-\frac{1}{6} t f\right) .
$$

Fig.2b:

$$
-\frac{C_{F}}{\varepsilon^{2}}\left[\frac{175}{72} C^{2}-\frac{55}{18} C t f+\frac{8}{9} t^{2} f^{2}\right]-\frac{C_{F}}{\varepsilon}\left[-\frac{2171}{432} C^{2}+\frac{527}{108} C t f+2 C_{F} t f-\frac{20}{27} t^{2} f^{2}\right] .
$$

Fig.2c:

$$
\begin{aligned}
-\frac{C_{F}^{2}}{\varepsilon^{3}}\left[\frac{1}{3} C-\frac{1}{6} C_{F}\right]+\frac{C_{F}^{2}}{\varepsilon^{2}}\left[3 C-\frac{7}{12} C_{F}\right. & \left.-\frac{2}{3} t f\right] \\
& -\frac{C_{F}^{2}}{\varepsilon}\left[\frac{91}{24} C-2 \zeta(3) C+\frac{1}{12} C_{F}-\frac{5}{6} t f\right] .
\end{aligned}
$$

Fig.2d: $\quad-\frac{2}{\varepsilon^{3}} C_{F}^{2} C$

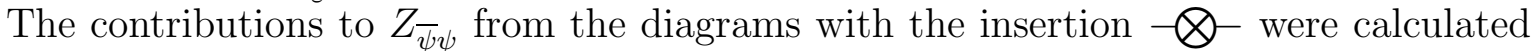
simultaneously with the diagrams shown in Fig.1 and Fig.2. In fact, for the fermion propagator instead of $\hat{p} / p^{2}$ we used $(\hat{p}+m) / p^{2}$, and after multiplication of all propagators we kept only the part without the mass term and the part linear in $m$. The coefficient in front of $m$ determines the contribution of the diagrams with the mass insertion. For example, from the diagram

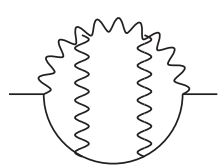


we get the following diagrams with the insertion:
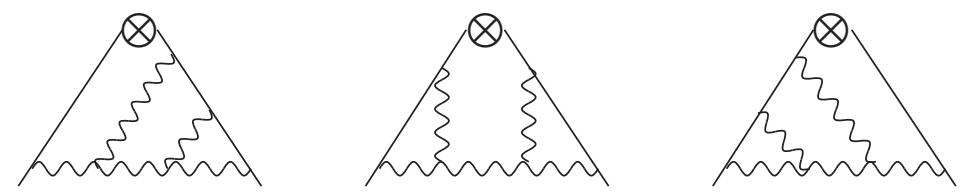

The contributions to $Z_{\bar{\psi} \psi}$ from different sets of diagrams $\left(K R^{\prime}\right)$

Fig.1:

$$
\begin{aligned}
& -\frac{C_{F}}{\varepsilon^{3}}\left[\frac{31}{3} C^{2}+\frac{50}{3} C C_{F}+8 C_{F}^{2}-4 C t f-\frac{8}{3} C_{F} t f\right] \\
& -\frac{C_{F}}{\varepsilon^{2}}\left[-\frac{181}{6} C^{2}-\frac{80}{3} C_{F} C+8 C_{F}^{2}+\frac{34}{3} C t f+\frac{8}{3} C_{F} t f\right] \\
& -\frac{C_{F}}{\varepsilon}\left[\frac{181}{4} C^{2}-\frac{145}{3} C C_{F}+17 C_{F}^{2}-13 C t f+\frac{20}{3} C_{F} t f\right] \\
& -\frac{C_{F}}{\varepsilon} \zeta(3)\left[-\frac{3}{2} C^{2}+20 C_{F} C-16 C_{F}^{2}-8 C t f\right] .
\end{aligned}
$$

Fig.2a:

$$
-\frac{C_{F}^{2}}{\varepsilon^{3}}\left[\frac{10}{3}-\frac{8}{3} t f\right]-\frac{C_{F}^{2}}{\varepsilon^{2}}\left[-\frac{17}{3} C+4 t f\right]-\frac{C_{F}^{2}}{\varepsilon}\left[-C+\frac{4}{3} t f\right] .
$$

Fig.2b:

$$
\begin{aligned}
& -\frac{C_{F}}{\varepsilon^{3}}\left[\frac{175}{36} C^{2}-\frac{55}{9} C t f+\frac{16}{9} t^{2} f^{2}\right] \\
& -\frac{C_{F}}{\varepsilon^{2}}\left[-\frac{337}{27} C^{2}+\frac{346}{27} C t f+4 C_{F} t f-\frac{64}{27} t^{2} f^{2}\right] \\
& -\frac{C_{F}}{\varepsilon}\left[\frac{18685}{1296} C^{2}-\frac{1915}{324} C t f-17 C_{F} t f-\frac{80}{81} t^{2} f^{2}\right] \\
& -\frac{C_{F}}{\varepsilon} \zeta(3)\left[-C^{2}-8 C t f+16 C_{F} t f\right]
\end{aligned}
$$

Fig.2c:

$$
\begin{aligned}
& -\frac{C_{F}^{2}}{\varepsilon^{3}}\left[6 C+\frac{8}{3} C_{F}-\frac{8}{3} t f\right]-\frac{C_{F}^{2}}{\varepsilon^{2}}\left[-17 C-10 C_{F}+4 t f\right] \\
& -\frac{C_{F}^{2}}{\varepsilon}\left[\frac{80}{3} C+5 C_{F}-\frac{16}{3} t f-16 \zeta(3) C+16 \zeta(3) C_{F}\right]
\end{aligned}
$$

Fig.2d: 0 . 


\section{References}

[1] Shirkov D. V. Mass Dependences In Renormalization Group Solutions // Theor. Math. Phys. 1982. V.49. P.1039-1043. [ Teor. Mat. Fiz. 1981. V.49. P.291];

Shirkov D. V. Threshold Effects At Two Loop Level And Parametrization Of The Real Qcd// Sov. J. Nucl. Phys. 1981. V. 34. P.300, [ Yad. Fiz. 1981. V.34. P.541-545].

[2] Kataev A.L.,Krasnikov N.V., Pivovarov A.A. The Use Of The Finite Energetic Sum Rules For The Calculation Of The Light Quark Masses// Phys. Lett. B. 1983. V.123. P.93-97.

[3] Nanopoulos D.V., Ross D.A. m(b)/m(tau): A sensitive flavor meter// Phys. Lett. B. 1982. V.108. P.351-354.

[4] Vladimirov A.A., Shirkov D.V. The Renormalization Group And Ultraviolet Asymptotics// Sov. Phys. Usp. 1979. V.22. P.860-878; [ Usp. Fiz. Nauk 1979. V.129. P.407].

[5] 't Hooft G. Dimensional regularization and the renormalization group// Nucl. Phys. B. 1973. V.61. P.455-468.

[6] Caswell W.E., Wilczek F. On the Gauge Dependence of Renormalization Group Parameters// Phys. Lett. B. 1974. V.49. P.291-292;

Banyai L., Marculescu S., Vescan T. Again on the Gauge Dependence of Renormalization Group Parameters// Lett. Nuovo Cim. 1974. V.11. P.151-155.

[7] Collins J.C., Macfarlane A.J. New methods for the renormalization group// Phys. Rev. D. 1974. V.10. P.1201-1212.

[8] Tarrach R. The Pole Mass in Perturbative QCD// Nucl. Phys. B. 1981. V.183. P.384-396;

Nachtmann O., Wetzel W. The Beta Function For Effective Quark Masses To Two Loops In Qcd// Nucl. Phys. B. 1981. V.187. P.333-342.

[9] Vladimirov A.A. Methods of Multiloop Calculations and the Renormalization Group Analysis of $\phi^{4}$ Theory// Theor. Math. Phys. 1979. V.36. P.732-737, [ Teor. Mat. Fiz. 1978. V.36. P.271-278].

[10] Strubbe H. Manual for Schoonschip: A CDC 6000 / 7000 program for symbolic evaluation of algebraic expressions// Comput. Phys. Commun. 1974. V.8. P.1-30.

[11] Espriu D., Palanques-Mestre A., Pascual P., Tarrach R., The Gamma Function In The 1/n-f Expansion// Z. Phys. C. 1982. V.13. P.153-156.

[12] Tarasov O.V., Vladimirov A.A., Phys.Part.Nucl. 2013. V.44. P.791-802;

Tarasov O.V., Vladimirov A.A., Three-loop calculations in non-Abelian gauge theories. JINR Commun. E2-80-483. Dubna. 1980. 24 p.

-arXiv:1301.5645 [hep-ph]. 